\title{
Tendencias de la personalidad y síntomas somáticos
}

Trends of the personality and somatic symptoms

\author{
José Moral de la Rubia \\ Universidad Autónoma de Nuevo León \\ Martha Adelina Torres Muñoz \\ José Luis Valdez Medina \\ Norma Ivonne González-Arratia López-Fuentes \\ Universidad Autónoma del Estado de México
}

\begin{abstract}
Resumen
Esta investigación tuvo por objetivo averiguar si hay diferencias en la manifestación de síntomas somáticos, tomando en cuenta el tipo de tendencia de personalidad [ego(+) o ego(-)] y el género. Se aplicó el Cuestionario de Tendencias de la Personalidad (CTP; Valdez, 2009) y la Escala de Síntomas Somáticos (ESS-R; Sandín \& Chorot, 1995). Los análisis de datos fueron hechos sobre una muestra no probabilística intencional de 128 adultos (70 hombres y 68 mujeres), de los cuales 64 pertenecían al tipo ego(+) y 64 al tipo ego(-). Se encontraron diferencias significativas por estilos de personalidad. Los participantes de tipo ego(-) fueron más sintomáticos, sufriendo más síntomas inmunológicos, gastrointestinales, alérgicos, hipertensivos y renales. Con base en la interrelación dentro de cada muestra de tipo de personalidad, los síntomas que involucran al sistema inmune se definieron más en el tipo ego(-) y los músculo-esqueléticos y genitourinarios en el tipo ego(+). También hubo diferencias por género, pero sin interacción con el tipo de personalidad. Las mujeres fueron más sintomáticas que los hombres. Así los tipos de personalidad y el género, como factores independientes, permiten considerar estrategias diferenciales de intervención en síntomas somáticos.
\end{abstract}

Palabras clave: Síntomas somáticos, personalidad, salud, género, adultos.

Nota del Autor:

José Moral de la Rubia, Facultad de Psicología, Universidad Autónoma de Nuevo León; Martha Adelina Torres Muñoz, Facultad de Ciencias de la Conducta, Universidad Autónoma del Estado de México; José Luis Valdez Medina, Facultad de Ciencias de la Conducta, Universidad Autónoma del Estado de México; Norma Ivonne González-Arratia López-Fuentes, Facultad de Ciencias de la Conducta, Universidad Autónoma del Estado de México.

La correspondencia en relación con este artículo debe dirigirse a José Moral de la Rubia, Facultad de Psicología, Universidad Autónoma de Nuevo León, c/Dr. Carlos Canseco 110, Col. Mitras Centro, C.P. 64460, Monterrey, Nuevo León, México.

Correo electrónico: jose_moral@ hotmail.com 


\section{Abstract}

The aims of this investigation were to find out differences in the somatic symptom display, taking into account the type of personality trend [ego(+) or ego(-)] and gender. We administered the Personality Trend Questionnaire (CTP; Valdez, 2009) and the Somatic Symptoms Scale (ESS-R; Sandín \& Chorot, 1995). Data analyses were conducted to the responses of a non probabilistic intentional sample of one 128 adults (60 and 68 women), of whom 64 corresponded to the ego(+) type and 64 to the type ego(-). Significant differences were found by personality styles. The type ego(-) participants were more symptomatic, and manifested more immunologic, gastrointestinal, allergic, hypertensive and renal symptoms. Based on the interrelationships within each personality type sample, the symptoms that involved the immunological system were more prevalent in the type ego(-), while the muscular-skeletal and genitourinary symptoms prevailed in the type ego(+). Differences by gender were also obtained, but they did not interact with the type of personality. Women were more symptomatic than men. Thus, the types of personality and gender allow considering differential strategies in somatic symptom intervention.

Keywords: Somatic symptoms, personality, health, gender, adults.

La somatización abarca un amplio rango de fenómenos clínicos y una variedad de procesos psicosociales (Lipowski, 1988). Estos conducen a la persona a buscar ayuda médica por malestares corporales para los cuales no se hallan causas orgánicas, a pesar de la insistencia del paciente en tal etiología. Según Vallejo (2003), el término somatización ha sido utilizado clásicamente para describir un proceso subconsciente por el que el individuo traduce su malestar emocional en quejas somáticas.

Desde esta conceptualización aparece una serie de síntomas, o signos físicos, de intensidad suficiente para ser considerados clínicamente, y para los que no existe una justificación médica. A éstos se les supone un sustrato psicológico donde se incluyen las emociones negativas (ansiedad, depresión o ira), conflictos (de dependencia no resuelta o de hostilidad reprimida), y rasgos de personalidad (alexitimia, neuroticismo, dependencia o irascibilidad).

Lobo, García, Campos, Marcos y Pérez (1996) informaban que en población española, la prevalencia anual de quejas no relacionadas con alguna enfermedad médica conocida se ubicó entre el $25 \%$ y $30 \%$ por ciento. Por otra parte, se indicó que entre el $60 \%$ y $80 \%$ por ciento de la población general padeció en algún momento de su vida un síntoma de estas características, de al menos una semana de duración (Woolfolk \& Allen, 2007). En la población infantil se encontraron síntomas somáticos recurrentes en el $11 \%$ de las niñas y en el cuatro por ciento de los niños entre 12 y 16 años de edad (Fritz, Fritsch, \& Hagino, 1997). Estas somatizaciones producen un deterioro en el funcionamiento y la calidad de vida de los pobladores. También se les asocia con el aumento de gastos y utilización de recursos sanitarios, como resultado de visitas médicas excesivas, pruebas diagnósticas, prescripciones terapéuticas y procedimientos quirúrgicos innecesarios (Hiller, Fichter, \& Rief, 2003).

\section{Personalidad: el modelo de las 16 tendencias y dos estilos de Valdez}

Se puede considerar que la personalidad está compuesta por dos grupos de variables con las que se nace y que posteriormente se van reinterpretando, o asignándoles significado en la síntesis que el individuo construye como su identidad. Por una parte estarían las variables biológicas, genéticas, hereditarias, físicas u orgánicas, entre estas se puede mencionar el aspecto físico, el género y la edad o etapa de desarrollo en la que se encuentre cada individuo. 
Por otra parte, estarían las de tipo psicosociocultural. Un ejemplo son las que hacen refe-rencia a la experiencia que va teniendo cada persona, la cultura en la que ha aprendido a vivir y el nivel socioeconómico. También la familia, el tipo de pares y los demás grupos de referencia o de pertenencia a los que se afilia cada individuo a lo largo de su vida (Hernández, 2000).

Valdez (2009), en su teoría de la Paz o Equilibrio, plantea que cada individuo tiene diferentes formas de enfrentarse a su medio, dependiendo de sus características de personalidad. Las tendencias de personalidad se conforman a partir de la aplicación, evaluación e interpretación de los resultados obtenidos de la utilización que las personas hacen de las dualidades o estrategias naturales de enfrentamiento. Dichas tendencias son definidas como estrategias de obtención de recursos o satisfactores para cubrir las necesidades o carencias y para evitar el miedo, y alcanzar el equilibrio o la paz.

Tales estrategias hacen usos de comportamientos muy específicos y de carácter natural o biológico como son: competir (ganar o perder), comparación (alta o baja jerarquía) y aceptar o rechazar (que puede llevar a la tolerancia y/o el control). Del resultado que se obtenga con estas estrategias cada uno va construyendo y desarrollando su propia tendencia de personalidad.

Los seres humanos tienden a construir y desarrollar a lo largo de sus vidas dos tipos básicos de tendencias de personalidad. La primera es fuerte o sana (ego cero); por sus características se ubican en un estado de seguridad, protección, confianza, paz o equilibrio, que, entre otras cosas, permiten conocerse a sí mismos de forma plena y certera.

La segunda es la tendencia de personalidad débil o en conflicto [ego(+) o ego(-)] regularmente se presenta en todas aquellas personas quienes, desde su infancia, y con mucha frecuencia, han tenido que tolerar y controlar, o bien mentir, aparentar, engañar, fingir, ocultar o aparentar ante sí mismos y ante los demás, como medidas de protección ante el miedo que se tiene por el rechazo o por el castigo, que no está dispuesto a aceptar o que se quiere evitar (Valdez, 2009).
Muchas veces el individuo, al poseer una tendencia de personalidad débil $[e g o(+) \quad 0$ ego(-)], presenta formas de adaptarse al medio y obtener recursos o satisfactores que son inadecuados. Probablemente esas formas de adaptarse tengan que ver con una tendencia a presentar ciertos síntomas somáticos. El tipo de personalidad ego(+) tiende a la ira, la confrontación y la competencia; así puede ser más susceptible a problemas cardiovasculares. El tipo ego(-) es más dependiente y pasivo, por lo que sufre más ansiedad e indefensión, padeciendo más trastornos relacionados con el sistema inmune y gastrointestinal, además de ser más sintomático o al menos buscan ayuda médica con más frecuencia (Valdez, 2009).

Valdez distingue 16 tendencias de personalidad que se conforman a partir del uso que se hace de las tolerancias individuales y sociales, así como a los controles individuales y sociales. Estas 16 tendencias permiten describir los estilos de personalidad ego(+) y al ego(-). El estilo ego cero es un tipo ideal y se manifiesta en ausencia de estas categorías descriptivas de aspectos desadaptativos. A continuación se enumera cada tendencia y se relaciona con su estilo correspondiente: 1) perfeccionista $(e g o+)$, 2) incomprendido social (ego -), 3) dejado (ego-), 4), baja autoestima (ego -), 5) desadaptado (ego -), 6) impositivo (ego +), 7) manipulador $($ ego +$), 8)$ incomprendido individual (ego -), 9) masoquista (ego -), 10) sádico (ego +), 11) fracasado (ego -), 12) ególatra (ego +), 13) autoritario (ego +), 14) sumiso (ego -), 15) reprimido (ego -) y 16) impulsivo $($ ego +$)$.

Desde estas bases teóricas se realizó una investigación para saber si existen diferencias entre las dos tendencias de las personalidades débiles $[e g o(+)$ o ego(-)] en relación con ciertos síntomas somáticos, aparte de estudiar las diferencias de género.

Se espera que el tipo de personalidad ego(-) y las mujeres sean más sintomáticas y con mayor definición de síntomas inmunológicos, al ser más proclives a la indefensión. Los estresores persistentes que no se afrontan con eficacia conducen a un estado de estrés crónico que suele generar manifestaciones diversas de 
desbalance vegetativo; y finalmente este estado conduce a otro de indefensión donde el sistema inmunológico muestra claros signos de debilitamiento por efecto de las hormones del estrés (Cardenal, Ortiz, \& Martín, 2008; Davis, 2009; Folkmann, 2011; Lazarus, 1999). Asimismo, se pronostican más síntomas gastrointestinales en la personalidad tipo ego(-) y cardiovasculares en la personalidad tipo ego(+) por internalización de la ira ante los conflictos y frustraciones en el primer tipo y externalización en el segundo, como reflejan distintos estudios de psicología de la salud (Eysenck, 2000; Kassinove, 1995; Moral, González, \& Landero, 2011; Spielberger \& Moscoso, 1996).

\section{Método}

\section{Participantes}

Se trabajó con una muestra no probabilística intencional de 128 adultos, divididas en 60 hombres y 68 mujeres, de los cuales 64 pertenecían a las tendencias de personalidad ego(+) (30 hombres y 30 mujeres) y 64 a las tendencias de personalidad ego(-) (32 hombres y 32 mujeres), siendo la frecuencia de género estadísticamente equivalente por la prueba binomial $(p=.54)$. Las edades variaban de 18 a 50 años con una media de 31 años. La escolaridad promedio fue de nueve punto un años (secundaria terminada). El 54\% de los encuestados eran casados, $15 \%$ divorciados o separados y $31 \%$ solteros.

\section{Instrumentos}

El Cuestionario de Tendencias de Personalidad (CTP) de Valdéz (2009) se conforma de cuatro ítems bipolares que se refieren a las tolerancias individuales (ítem A-B) y sociales (ítem C-D), así como a los controles individuales (ítem E-F) y sociales (ítem G-H) que todos los seres humanos utilizan para intentar conseguir los recursos o satisfactores que cubran sus necesidades o carencias, evitar el miedo al rechazo y el castigo, así como conseguir el equilibrio y paz personal. Con base en estos cuatro ítems se pueden obtener dieciséis tipos de tendencias de la personalidad, pertenecientes a dos tipos de tendencia de personalidad ego(+) o ego(-), reflejando la ausencia de estas tendencias un tipo ego(0). Se observa una coincidencia del setenta y cinco al ochenta por ciento entre el instrumento de autoinforme y observadores expertos al momento de clasificar a los participantes en estos 16 tipos de personalidad (Valdez, 2009).

La Escala Revisada de Síntomas Somáticos (ESS-R) de Sandin y Chorot (1995) es un cuestionario, tipo autoinforme, que permite evaluar los diferentes tipos de manifestaciones somáticas relacionadas con la salud. Consta de 80 ítems con un rango de valoración de frecuencia de 5 puntos: 0 (nunca), 1 (raras veces), 2 (algunas veces), 3 (frecuentemente) y 4 (casi siempre). En el mismo se incluyen 10 ítems adicionales que hacen referencia a los dolores o molestias relacionados con el período menstrual y que, por tanto, sólo son contestados por las mujeres, los cuales no se analizan en este estudio. El participante evalúa la frecuencia de los síntomas en el último año. Los órganos y sistemas evaluados son los siguientes: (1) inmunológico general, (2) cardiovascular, (3) respiratorio, (4) gastrointestinal, (5) neurosensorial, (6) dermatológico, (7) músculo-esquelético, (8) genitourinario y (9) reproductor femenino. La consistencia interna de la escala es mayor a .80 y la de sus factores varía de .70 a .84. Resulta estable a las dos semanas $(r=.71)$ y correlaciona, según las expectativas, con diversos factores de riesgo y protección (Sandin \& Chorot, 1995).

\section{Procedimiento}

Las aplicaciones se llevaron a cabo de manera individual, con una duración de entre 20 y 30 minutos, en forma de cuestionario autoaplicado, en la consulta externa del Sistema de Salud Estatal de la Ciudad de Toluca, Estado de México. Inicialmente se aplicó el instrumento de tendencias de la personalidad (Valdez, 2009), para clasificar a los participantes [ego(0), ego(+) y ego(-)] con una confiabilidad interjuez de $80 \%$. Luego se procedió a aplicar la escala de síntomas somáticos de Sandín y Chorot (1995). En un principio se aplicaron ambos instrumentos 
a 100 personas (CTP y ESS-R), 50 hombres y 50 mujeres, pero se obtuvo un porcentaje mayor de personalidad tipo ego(-) (64 de 100, 34 mujeres y 30 hombres) que del tipo ego(+) (36 de 100, 16 mujeres y 20 hombres), por lo que se cambió de estrategia. De forma intencional se buscaron personas que tuvieran el tipo ego(+), aplicando sólo el cuestionario CTP, por lo tanto necesitábamos encontrar 28 personas con tendencia ego(+) que desearan participar. En esta segunda etapa del muestreo, sólo cuando el cuestionario CTP mostraba tendencia ego(+) se aplicaba la segunda escala (ESS-R), en caso contrario se descartaba el dato para el estudio. Además, se procuró mantener el equilibrio de género, a pesar de que la participación voluntaria y disponibilidad era mayor en mujeres. De este modo se logró que la muestra quedara equilibrada entre los tipos ego(-) y ego(+), 64 participantes en cada grupo, y fuese equivalente en la integración del género, es decir, sin sesgo en estas dos variables a la hora de hacer los contrastes por síntomas somáticos.

\section{Análisis estadísticos}

Para la comparación de medias en la frecuencia de síntomas y de los factores de síntomas, entre los dos tipos de tendencias de personalidad $[e g o(+)$ o ego(-)] y el género, se empleó la prueba $t$ de Student para muestras independientes; y para estimar la interacción entre ambas variables se usó un análisis de varianza de dos vías de factores fijos e independientes. Además, se factorizó la frecuencia de síntomas en cada submuestra de tendencia de personalidad para explorar las dimensiones subyacentes diferenciales. Se empleó el análisis de componentes principales y una rotación ortogonal por el método Varimax. Se considera la saturación más alta y mayor a .40 de cada síntoma en la matriz factorial rotada para la definición e interpretación de los factores. El número de factores se fija por el criterio de Cattell (punto de inflexión de la curva de sedimentación). Las dimensiones de los síntomas en la muestra conjunta también se establecen por este procedimiento de análisis factorial exploratorio. Las puntuaciones de los factores de síntomas se obtienen por el método de Anderson-Rubin (Moral, 2006). El nivel de significación para el rechazo de la hipótesis nula se fija en $p<.05$. Los cálculos se realizaron con el programa estadístico SPSS versión 16.

\section{Resultados}

\section{Descripción de los síntomas en la muestra}

De acuerdo a la media aritmética, los síntomas que más aparecen, con un promedio de algunas veces durante el último año, son: malestar en el estómago, dolores de espalda, dolores de cabeza, catarro, gripe, dolor de estómago, gastritis, estornudos repetitivos, dolor de garganta, dolores musculares y acidez de estómago (véase tabla 1).

\section{Tabla 1}

Medias y desviaciones estándar de síntomas somáticos en la muestra total $(N=128)$

\begin{tabular}{lcccc}
\hline \multicolumn{1}{c}{ Síntomas Somáticos } & Min & Max & M & DE \\
\hline Malestar en el estómago & 1 & 5 & 2.65 & 1.12 \\
Dolores de espalda & 1 & 5 & 2.43 & 1.21 \\
Dolores de cabeza & 1 & 5 & 2.28 & 1.16 \\
Catarro & 1 & 5 & 2.27 & 0.95 \\
Gripe & 1 & 5 & 2.23 & 0.93 \\
Dolor de estómago & 1 & 5 & 2.20 & 1.03 \\
Gastritis & 1 & 5 & 2.13 & 1.22 \\
Estornudos repetitivos & 1 & 5 & 2.10 & 1.22 \\
Dolor de garganta & 1 & 5 & 2.05 & 0.97 \\
Dolores musculares & 1 & 5 & 2.05 & 1.06 \\
Acidez de estómago & 1 & 5 & 2.02 & 1.08 \\
\hline
\end{tabular}




\section{Diferencias por género}

Los hombres tienden más que las mujeres a presentar síntomas como diarrea y vómitos. Las mujeres son más propensas a presentar síntomas como dolor de cabeza, dolores en la nuca o zona cervical, colitis, cansancio o fatiga sin causa aparente, calambres o dolor en las piernas, estreñimiento o dificultades para defecar, sensación de oleadas de frío o calor y prurito en los ojos de tipo alérgico (véase tabla 2).

\section{Diferencias por tipo de personalidad}

Los participantes con tendencia de personalidad denominada como ego(-) padecen significativamente más síntomas somáticos que los participantes con tendencia ego(+); siendo nueve los síntomas diferenciales: molestias para orinar, dificultad para orinar, dolor de estómago, prurito o escozor en la zona genital que incitan a rascarse, molestias o dolor en riñones, molestias en zonas genitales, sarpullido en la piel nuevo o que reaparece, diarrea y torpeza en las manos (véase tabla 3). Ninguna interacción entre el género y el tipo de personalidad resulta significativa, al comparar las medias de frecuencias de los síntomas por análisis de varianza de dos vías.

Al factorizar en la muestra conjunta se definen 24 factores por el criterio de Kaiser (autovalores iniciales mayores a uno) que explican el $77.04 \%$ de la varianza total. Se pueden reducir a ocho por el criterio de Cattell, explicándose el $47.25 \%$ de la varianza total.

Tabla 2

Diferencia de medias de frecuencia de síntomas somáticos por género

\begin{tabular}{|c|c|c|c|c|c|c|}
\hline \multirow[t]{2}{*}{ Síntomas somáticos } & \multirow[t]{2}{*}{$\mathrm{t}$} & \multirow[t]{2}{*}{$\mathrm{p}$} & \multicolumn{2}{|c|}{$\begin{array}{c}\text { Hombres } \\
(\mathrm{n}=60)\end{array}$} & \multicolumn{2}{|c|}{$\begin{array}{l}\text { Mujeres } \\
(\mathrm{n}=68)\end{array}$} \\
\hline & & & M & $\mathrm{DE}$ & M & $\mathrm{DE}$ \\
\hline Dolor de cabeza & 4.40 & .00 & 1.83 & 0.94 & 2.68 & 7.19 \\
\hline Dolores en la nuca o zona cervical & 3.28 & .00 & 1.57 & 0.90 & 2.16 & 1.31 \\
\hline Diarrea & 3.09 & .00 & 1.92 & 0.92 & 1.47 & 0.70 \\
\hline Colitis & 2.77 & .01 & 1.48 & 0.98 & 2.06 & 1.31 \\
\hline Cansancio o fatiga sin causa aparente & 2.51 & .01 & 1.45 & 0.76 & 1.87 & 0.06 \\
\hline Calambres o dolor en las piernas & 2.39 & .02 & 1.65 & 0.86 & 2.10 & 1.22 \\
\hline $\begin{array}{l}\text { Estreñimiento o dificultades para } \\
\text { defecar }\end{array}$ & 2.37 & .02 & 1.52 & 0.74 & 1.96 & 1.26 \\
\hline Vómitos & 2.33 & .02 & 1.43 & 0.67 & 1.19 & 0.49 \\
\hline Sensación de oleadas de frío o calor & 2.19 & .03 & 1.63 & 0.78 & 1.99 & 1.00 \\
\hline Picor en los ojos de tipo alérgico & 2.18 & .03 & 1.33 & 0.70 & 1.69 & 1.08 \\
\hline
\end{tabular}




\section{Tabla 3}

Diferencia de medias de frecuencia de síntomas somáticos por tipo de tendencia de personalidad

\begin{tabular}{|c|c|c|c|c|c|c|}
\hline \multirow[t]{2}{*}{ Síntomas somáticos } & \multirow[t]{2}{*}{$\mathrm{t}$} & \multirow[t]{2}{*}{$\mathrm{p}$} & \multicolumn{2}{|c|}{$\begin{array}{l}\text { Ego(+) } \\
\mathrm{n}=64\end{array}$} & \multicolumn{2}{|c|}{$\begin{array}{l}\text { Ego(-) } \\
\mathrm{n}=64\end{array}$} \\
\hline & & & M & DE & M & $\mathrm{DE}$ \\
\hline Molestias para orinar & 4.31 & .00 & 1.05 & 0.21 & 1.42 & 0.66 \\
\hline Dificultad para orinar & 2.79 & .01 & 1.08 & 0.32 & 1.38 & 0.78 \\
\hline Dolor de estómago & 2.26 & .02 & 2.00 & 0.99 & 2.41 & 1.03 \\
\hline $\begin{array}{l}\text { Sensaciones de picor o escozor en la zona } \\
\text { genital que incitan a rascarse }\end{array}$ & 2.24 & .03 & 1.20 & 0.44 & 1.44 & 0.71 \\
\hline Molestias o dolor en riñones & 2.21 & .03 & 1.42 & 0.83 & 1.78 & 1.00 \\
\hline Molestias en zonas genitales & 2.21 & .03 & 1.09 & 0.34 & 1.31 & 0.71 \\
\hline Granos en la piel nuevos o que reaparecen & 2.19 & .03 & 1.39 & 0.74 & 1.72 & 0.93 \\
\hline Diarrea & 2.02 & .04 & 1.53 & 0.79 & 1.83 & 0.86 \\
\hline Torpeza en las manos & 1.99 & .04 & 1.11 & 0.44 & 1.30 & 0.60 \\
\hline
\end{tabular}

El primer componente factorial, integrado por nueve síntomas somáticos con cargas factoriales mayores a .40 (molestias en zonas genitales, dolor en zonas genitales, visión doble o deformada, sensaciones de adormecimiento en brazos o piernas, calambres o dolor en las piernas, cansancio o fatiga sin causa aparente, sensaciones de opresión o molestias en el pecho, escozor o malestar al orinar o después de orinar, así como fibroma o tumor nuevos que reaparecen) y consistencia interna alta $(\alpha=.80)$, se puede denominar: de síntomas genitourinarios y pseudoneurológicos, es decir, de naturaleza histérico-conversiva.

El segundo, definido por ocho síntomas somáticos con cargas factoriales mayores a .40 (colitis, constipado o cogestión de tipo alérgico, síntomas de conjuntivitis alérgica, prurito nasal de tipo alérgico, gastritis, acidez de estómago, prurito ocular de tipo alérgico, así como estreñimiento o dificultades para defecar) y consistencia interna alta $(\alpha=.82)$, se puede denominar: de síntomas gastrointestinales y alérgicos.

El tercero, compuesto por doce síntomas somáticos con cargas factoriales mayores a .40 (tos acompañada de flemas, prurito o escozor en la piel, fiebre, dolor de garganta, molestias o dolor en riñones no debidas a ejercicio, erupciones en la piel, ligero dolor en el pecho al iniciar algún esfuerzo físico, respiración dificultosa acompañada de tos, dolor en el pecho, sarpullido cutáneo nuevo o que reaparece, diarrea, así como contracciones o sacudidas musculares) y consistencia interna alta $(\alpha=.82)$, se puede denominar: de síntomas gripales, cata-rrales o alérgicos.

El cuarto, conformado por nueve síntomas somáticos con cargas factoriales mayores a .40 (inflamación de las articulaciones, dolor de articulaciones, dolores musculares, sensación de hormigueo en alguna parte del cuerpo, dificultades de coordinación, temblor muscular, dolores de espalda, visión borrosa, así como torpeza en las manos) y consistencia interna alta $(\alpha=.80)$, se puede denominar: de dolores musculo-esqueléticos y en extremidades.

El quinto, configurado por nueve síntomas somáticos con cargas factoriales mayores a .40 (dificultad para respirar durante el esfuerzo, taquicardias, palpitaciones, molestias 0 sensación de malestar en el estómago, estado general de baja energía, dolores abdominales, dolores de cabeza con sensación de tirantez o tensión, dolor de estómago, así como mareos) y consistencia interna alta $(\alpha=.79)$, se puede denominar: de síntomas cardiovasculares.

El sexto, definido por siete síntomas somáticos con cargas factoriales mayores a .40 (necesidad de hacer dieta para reducir la tensión arterial, tensión arterial ligeramente alta, necesidad de tomar medicina para reducir la tensión arterial, sangre en la orina, molestias al orinar, respiración 
dificultosa acompañada de ruidos, así como dificultades para orinar) y consistencia interna ala $(\alpha=.79)$, se puede denominar: de hipertensión y problemas renales.

El séptimo, formado por seis síntomas somáticos con cargas factoriales mayores a .40 (nauseas o sensación de vómitos, sudoración excesiva, catarros o resfriados comunes, gripe, estornudos repetitivos, así como necesidad de orinar con frecuencia) y consistencia interna alta $(\alpha=.70)$, se puede denominar: de síntomas catarrales, gripales o infecciosos.

El octavo, compuesto por seis síntomas somáticos con cargas factoriales mayores o iguales a .40 (dolor en el pecho acompañado de fatiga y respiración dificultosa, sensación de ahogo o respiración dificultosa tras emociones fuertes, respiración dificultosa o molesta, sensaciones de ahogo o falta de respiración, tos repetitiva, así como sensación de falta de aire) y consistencia interna alta $(\alpha=.72)$, se puede denominar: de síntomas respiratorios.

Todos ellos tienen valores de consistencia interna alta $(\alpha>.70)$. Sólo la distribución del séptimo componente de síntomas gripales, catarrales o infecciosos se ajusta a una curva normal $(Z K-S=1.08, p=.20)$ (véase tabla 4 ).

Se obtienen las puntuaciones factoriales por el método de Anderson-Rubin y se contrastan las medias de los ocho componentes entre los dos tipos de tendencia de personalidad. Las alteraciones catarrales, gripales o alérgicas ( $t$ $(125)=-3.10, p<.01)$, así como los problemas de hipertensión y renales $(\mathrm{t}(80.56)=-2.52, \mathrm{p}=$ .01) son reportados con más frecuencia por el tipo ego(-); a su vez, los síntomas genitourina-rios y pseudoneurológicos o histérico-conversivos se aproximan a la significación estadística (t (125) $=-1.87, p=.06)$, con mayor promedio en el tipo ego(-) (véase tabla 5).

Tabla 4

Matriz factorial rotada en la muestra conjunta $(N=128)$

\begin{tabular}{|c|c|c|c|c|c|c|c|c|}
\hline \multirow{2}{*}{$\begin{array}{l}\text { Síntomas } \\
\text { somáticos }\end{array}$} & \multicolumn{8}{|c|}{ Componentes } \\
\hline & $\mathrm{C} 1$ & $\mathrm{C} 2$ & C3 & $\mathrm{C} 4$ & C5 & C6 & $\mathrm{C} 7$ & $\mathrm{C} 8$ \\
\hline $\begin{array}{c}\text { Molestias en zonas } \\
\text { genitales }\end{array}$ & .83 & & & & & & & \\
\hline Dolor en zonas genitales & .71 & & & & & & & \\
\hline Visión doble & .59 & & & & & & & \\
\hline $\begin{array}{l}\text { Adormecimiento de } \\
\text { extremidades }\end{array}$ & .56 & & & & & & & \\
\hline Calambres & .54 & & & & & & & \\
\hline Fatiga & .52 & & & & & & & \\
\hline Opresión en el pecho & .47 & & & & & & & \\
\hline Malestar al orinar & .46 & & & & & & & \\
\hline Tumores & .44 & & & & & & & \\
\hline Colitis & & .69 & & & & & & \\
\hline Congestión alérgica & & .66 & & & & & & \\
\hline Conjuntivitis & & .66 & & & & & & \\
\hline Picor de nariz por alergia & & .62 & & & & & & \\
\hline Gastritis & & .61 & & & & & & \\
\hline Acidez estomacal & & .59 & & & & & & \\
\hline Picor de ojos por alergia & & .58 & & & & & & \\
\hline
\end{tabular}


Tabla 4 (Cont.)

Matriz factorial rotada en la muestra conjunta

\begin{tabular}{|c|c|c|c|c|c|c|c|c|}
\hline \multirow{2}{*}{$\begin{array}{l}\text { Síntomas } \\
\text { somáticos }\end{array}$} & \multicolumn{8}{|c|}{ Componentes } \\
\hline & $\mathrm{C} 1$ & $\mathrm{C} 2$ & $\mathrm{C3}$ & $\mathrm{C} 4$ & $\mathrm{C} 5$ & $\mathrm{C} 6$ & $\mathrm{C} 7$ & $\mathrm{C} 8$ \\
\hline Estreñimiento & & .56 & & & & & & \\
\hline Tos con flemas & & & .61 & & & & & \\
\hline Picor o escozor en la piel & & & .57 & & & & & \\
\hline Fiebre & & & .55 & & & & & \\
\hline Dolor de garganta & & & .53 & & & & & \\
\hline Molestias o dolor en riñones & .44 & & .52 & & & & & \\
\hline Salpullido & .42 & & .51 & & & & & \\
\hline $\begin{array}{c}\text { Dolor de pecho al iniciar } \\
\text { esfuerzo }\end{array}$ & & & .51 & & & & & \\
\hline Respiración difícil con tos & & & .50 & & & & & .43 \\
\hline Dolor de pecho & & & .50 & & & & & \\
\hline Granos & & & .47 & & & & & \\
\hline Diarrea & & & .44 & & & & & \\
\hline Contracciones musculares & & & .43 & & & & & \\
\hline Inflación de articulaciones & & & & .63 & & & & \\
\hline Dolor articulaciones & & & & .61 & & & & \\
\hline Debilidad muscular & & & & .59 & & & & \\
\hline Hormigueo & & & & .59 & & & & \\
\hline Problemas de coordinación & & & & .55 & & & & \\
\hline Temblor muscular & & & & .53 & & & & \\
\hline Dolor de espalda & & & & .47 & & & & \\
\hline Visión borrosa & & & & .45 & & & & \\
\hline Torpeza en las manos & & & & .43 & & .41 & & \\
\hline Dificultad para respirar & & & & & .76 & & & \\
\hline Taquicardia & & & & & .59 & .46 & & \\
\hline Palpitaciones & & & & & .56 & & & \\
\hline Malestar de estómago & & .43 & & & .55 & & & \\
\hline Baja energía & & & & & .48 & & & \\
\hline Dolor de abdomen & & .45 & & & .46 & & & \\
\hline Dolor cabeza & & & & & .45 & & & \\
\hline Dolor de estomago & & & & & .43 & & & \\
\hline Mareos & & & & & .42 & & & \\
\hline $\begin{array}{l}\text { Dieta para controlar la } \\
\text { tensión arterial }\end{array}$ & & & & & & .82 & & \\
\hline Tensión alta & & & & & & .80 & & \\
\hline Medicación para la tensión & & & & & & .66 & & \\
\hline
\end{tabular}


Tabla 4 (Cont.)

Matriz factorial rotada en la muestra conjunta

\begin{tabular}{|c|c|c|c|c|c|c|c|c|c|}
\hline \multirow{2}{*}{\multicolumn{2}{|c|}{$\begin{array}{l}\text { Síntomas } \\
\text { somáticos }\end{array}$}} & \multicolumn{8}{|c|}{ Componentes } \\
\hline & & C1 & $\mathrm{C} 2$ & $\mathrm{C} 3$ & $\mathrm{C} 4$ & C5 & $\mathrm{C} 6$ & $\mathrm{C7}$ & $\mathrm{C} 8$ \\
\hline \multicolumn{2}{|c|}{ Sangre en la orina } & & & & & & .60 & .40 & \\
\hline \multicolumn{2}{|c|}{ Molestias al orinar } & & & & & & .49 & & \\
\hline \multicolumn{2}{|c|}{ Ruidos al respirar } & & & & & & .47 & & \\
\hline \multicolumn{2}{|c|}{ Dificultad para orinar } & & & & & & .44 & & \\
\hline \multicolumn{2}{|c|}{ Nausea } & & & & & & & .60 & \\
\hline \multicolumn{2}{|c|}{ Sudor excesivo } & & & & & & & .58 & \\
\hline \multicolumn{2}{|c|}{ Catarro } & & & & & & & .53 & \\
\hline \multicolumn{2}{|c|}{ Gripe } & & & & & & & .49 & \\
\hline \multicolumn{2}{|c|}{ Estornudos } & & .40 & & & & & .45 & \\
\hline \multicolumn{2}{|c|}{$\begin{array}{l}\text { Necesidad de orinar } \\
\text { frecuente }\end{array}$} & & & & & & & .43 & \\
\hline \multicolumn{2}{|c|}{ Fatiga } & & & & & & & & .77 \\
\hline \multicolumn{2}{|c|}{$\begin{array}{c}\text { Ahogo tras emociones } \\
\text { fuertes }\end{array}$} & & & & & & & & .55 \\
\hline \multicolumn{2}{|c|}{ Respiración dificultosa } & & & & & & & .45 & .50 \\
\hline \multicolumn{2}{|c|}{ Sensaciones de ahogo } & .45 & & & & & & & .47 \\
\hline \multicolumn{2}{|c|}{ Tos repetitiva } & & & & & & & & .41 \\
\hline \multicolumn{2}{|c|}{ Falta de aire } & & & & & & & & .40 \\
\hline \multicolumn{2}{|c|}{ Autovalor } & 5.76 & 5.17 & 5.12 & 4.88 & 4.74 & 4.65 & 3.81 & 3.66 \\
\hline \multicolumn{2}{|c|}{$\%$ de varianza explica } & 7.20 & 6.46 & 6.40 & 6.11 & 5.93 & 5.81 & 4.76 & 4.58 \\
\hline \multicolumn{2}{|c|}{$\%$ acumulado } & 7.20 & 13.66 & 20.06 & 26.16 & 32.09 & 37.91 & 42.67 & 47.25 \\
\hline \multirow{2}{*}{$\begin{array}{l}\text { Consistencia } \\
\text { interna }\end{array}$} & No. Ítems & 9 & 8 & 12 & 9 & 9 & 7 & 6 & 6 \\
\hline & $\alpha$ & .80 & .82 & .82 & .80 & .79 & .79 & .70 & .72 \\
\hline \multirow{3}{*}{$\begin{array}{l}\text { Descriptivos } \\
\text { de la } \\
\text { distribución }\end{array}$} & $\mathrm{N}$ & 127 & 127 & 127 & 127 & 127 & 127 & 127 & 127 \\
\hline & $\mathrm{M}$ & 1 & 1 & 1 & 1 & 1 & 1 & 1 & 1 \\
\hline & $\mathrm{DE}$ & 0 & 0 & 0 & 0 & 0 & 0 & 0 & 0 \\
\hline \multirow{2}{*}{$\begin{array}{l}\text { Ajuste a la } \\
\text { normalidad }\end{array}$} & ZK-S & 1.88 & 2.07 & 1.54 & 2.10 & 1.51 & 2.51 & 1.08 & 1.61 \\
\hline & $\mathrm{P}$ & .00 & .00 & .20 & .00 & .02 & .00 & .02 & .01 \\
\hline
\end{tabular}

C1: Síntomas genitourinarios y pseudoneurológicos, C2: Síntomas gastrointestinales y alérgicos, C3: Síntomas gripales, catarrales o alérgicos, C4: Dolores musculo-esqueléticos y en extremidades, C5: Síntomas cardiovasculares, C6: Hipertensión y problemas renales, C7: Síntomas catarrales, gripales o infecciosos y C8: Síntomas respiratorios. Para definir a cada componente sólo se considera la carga más alta y mayor o igual a .40 de cada síntoma (en cursiva). 
Tabla 5

Diferencia de medias en los 8 factores entre los participantes con personalidad ego(-) y ego(+) en la muestra conjunta $(N=128)$

\begin{tabular}{lcccccc}
\hline \multicolumn{1}{c}{\begin{tabular}{c} 
Factores \\
\multicolumn{1}{c}{ de síntomas somáticos }
\end{tabular}} & \multicolumn{2}{c}{ Levene } & \multicolumn{3}{c}{ Student } & \begin{tabular}{c} 
Dif. \\
\cline { 2 - 6 }
\end{tabular} \\
\cline { 2 - 5 } & 3.16 & .08 & -1.87 & 125 & .06 & -0.33 \\
$\begin{array}{l}\text { Síntomas genitourinarios y } \\
\text { pseudoneurológicos }\end{array}$ & 0.90 & .34 & -0.29 & 125 & .77 & -0.05 \\
$\begin{array}{l}\text { Síntomas gastrointestinales } \\
\text { y alérgicos }\end{array}$ & 1.58 & .21 & -3.10 & 125 & .00 & -0.53 \\
$\begin{array}{l}\text { Síntomas catarrales, gripales } \\
\text { o alérgicos }\end{array}$ & 1.67 & .20 & 0.55 & 125 & .58 & 0.10 \\
$\begin{array}{l}\text { Dolores musculo- } \\
\text { esqueléticos }\end{array}$ & 4.74 & .03 & 1.09 & 114.84 & .28 & 0.19 \\
$\begin{array}{l}\text { Síntomas cardiovasculares } \\
\text { Problemas de hipertensión y }\end{array}$ & 11.13 & .00 & -2.52 & 80.56 & .01 & -0.44 \\
$\begin{array}{l}\text { renales } \\
\text { Síntomas catarrales, gripales }\end{array}$ & 0.75 & .39 & 1.19 & 125 & .23 & 0.21 \\
e infecciosos & 1.10 & .30 & 0.47 & 125 & .64 & 0.08 \\
\hline Síntomas respiratorios & & & & & & \\
\hline
\end{tabular}

\section{Agrupación de síntomas en cada tipo de personalidad}

Tras estudiar las diferencias de medias, se procedió a factorizar para observar cómo se agrupaban los ítems dentro de cada tendencia de personalidad.

Dentro de la submuestra de participantes con tendencia de personalidad tipo ego(-), se obtuvieron 23 factores por el criterio de Kaiser; sin embargo, con base en la claridad conceptual y el punto de inflexión de la curva de sedimentación de los autovalores (criterio de Cattell), se seleccionaron los once primeros que explican el $63.38 \%$ de la varianza total: (1) bajas defensas o problemas relacionados con tumores, (2) problemas gastrointestinales, (3) síntomas vestibulares o infecciosos con fiebre, (4) hipertensión arterial, (5) cardiorrespiratorios y estomacales, (6) dermatológicos y genitourinarios, (7) neurológicos o reumatoides, (8) cardiovasculares, (9) alergias, (10) infecciones en vías respiratorias altas y (11) problemas circulatorios y en la piel probablemente relacionados con exceso de grasas en la dieta (véase tabla 6).

Dentro de la submuestra de participantes con tendencia de personalidad tipo ego(+), se obtuvieron 22 factores por el criterio de Kaiser. Sin embargo, con base en la claridad conceptual y el criterio de Cattell, se seleccionaron los nueve primeros que explican el $58.39 \%$ de de la varianza total: (1) dolores músculo-esqueléticos y reumatoides, (2) síntomas genitourinarios y neurológicos, (3) del sistema respiratorio, (4) dermatológicos y vasovagales o vestibulares, (5) cansancio/baja energía, (6) síntomas alérgicos y estomacales, (7) síntomas causados por tumores, (8) síntomas relacionados con hiperactividad simpática y (9) hipertensión arterial (véase tabla 7). 
Tabla 6

Matriz factorial rotada en la muestra de participantes tipo Ego(-)

\begin{tabular}{|c|c|c|c|c|c|c|c|c|c|c|c|}
\hline Síntomas somáticos & C1 & $\mathrm{C} 2$ & C3 & $\mathrm{C} 4$ & $\mathrm{C} 5$ & $\mathrm{C} 6$ & $\mathrm{C} 7$ & C8 & C9 & $\mathrm{C} 10$ & C11 \\
\hline Molestias en zonas genitales & .86 & & & & & & & & & & \\
\hline Dolor en zonas genitales & .80 & & & & & & & & & & \\
\hline Molestias en oídos & .68 & & & & & & & & & & \\
\hline Calambres o dolor en las piernas & .63 & & & & & & & & & & \\
\hline $\begin{array}{c}\text { Cansancio o fatiga sin causa } \\
\text { aparente }\end{array}$ & .60 & & & & & & & & & & \\
\hline $\begin{array}{l}\text { Fibroma o tumor nuevos que } \\
\text { reaparecen }\end{array}$ & .59 & & & & & & & & & & \\
\hline Estado general de baja energía & .58 & & & & & & & & & & \\
\hline Sensaciones de ahogo & .53 & & & & & & & & & & \\
\hline Molestias o dolor en riñones & .50 & & & & & & & & & & \\
\hline Dolor en el pecho & .49 & & & & & & & & & & \\
\hline Estornudos repetitivos & .40 & & & & & & & & & & \\
\hline Colitis & & .79 & & & & & & & & & \\
\hline Acidez de estómago & & .79 & & & & & & & & & \\
\hline Gastritis & & .67 & & & & & & & & & \\
\hline Temblor muscular & & .64 & & & & & & & & & \\
\hline Tos repetitiva & & .57 & & & & & & & & & \\
\hline Dolor de estómago & & .56 & & & & & & & & & \\
\hline Estreñimiento & & .54 & & & & & & & & & \\
\hline Dolores abdominales & & .49 & & & & & & & & & \\
\hline Mareos & & & .76 & & & & & & & & \\
\hline Vértigos & & & .75 & & & & & & & & \\
\hline Respiración dificultosa con tos & & & .66 & & & & & & & & \\
\hline Escozor o picor en la piel & & & .64 & & & & & & & & \\
\hline Fiebre & & & .62 & & & & & & & & \\
\hline Erupciones en la piel & & & .54 & & & & & & & & \\
\hline $\begin{array}{c}\text { Medicina para reducir la tensión } \\
\text { arterial }\end{array}$ & & & & .84 & & & & & & & \\
\hline $\begin{array}{c}\text { Necesidad de dieta para tensión } \\
\text { arterial }\end{array}$ & & & & .80 & & & & & & & \\
\hline Tensión arterial ligeramente alta & & & & .74 & & & & & & & \\
\hline Sangre en la orina & & & & .72 & & & & & & & \\
\hline Taquicardia & & & & .64 & & & & & & & \\
\hline Dificultad para respirar en esfuerzo & & & & & .85 & & & & & & \\
\hline Respiración dificultosa o molesta & & & & & .78 & & & & & & \\
\hline Palpitaciones & & & & & .60 & & & & & & \\
\hline
\end{tabular}


Tabla 6. (Cont.)

Matriz factorial rotada en la muestra de participantes tipo Ego(-)

\begin{tabular}{|c|c|c|c|c|c|c|c|c|c|c|c|}
\hline Síntomas somáticos & $\mathrm{C} 1$ & $\mathrm{C} 2$ & C3 & C4 & C5 & C6 & $\mathrm{C} 7$ & C8 & C9 & C10 & C11 \\
\hline Malestar en el estómago & & & & & .57 & & & & & & \\
\hline Eccema & & & & & & .79 & & & & & \\
\hline Dificultades para orinar & & & & & & .78 & & & & & \\
\hline Molestias al orinar & & & & & & .56 & & & & & \\
\hline Dificultades de coordinación & & & & & & & .84 & & & & \\
\hline Torpeza en las manos & & & & & & & .71 & & & & \\
\hline $\begin{array}{l}\text { Sensación de inestabilidad en la } \\
\text { marcha }\end{array}$ & & & & & & & .59 & & & & \\
\hline Inflamación de articulaciones & & & & & & & .54 & & & & \\
\hline $\begin{array}{l}\text { Ligero dolor en el pecho al } \\
\text { esforzarse }\end{array}$ & & & & & & & & .75 & & & \\
\hline Dolor en el pecho & & & & & & & & .65 & & & \\
\hline Contracciones musculares & & & & & & & & .58 & & & \\
\hline Diarrea & & & & & & & & .44 & & & \\
\hline Fatiga & & & & & & & & .41 & & & \\
\hline Constipado o congestión alérgica & & & & & & & & & .83 & & \\
\hline Picores en la nariz de tipo alérgico & & & & & & & & & .81 & & \\
\hline Conjuntivitis alérgica & & & & & & & & & .45 & & \\
\hline Dolor de espalda & & & & & & & & & .44 & & \\
\hline Catarros o resfriados comunes & & & & & & & & & & .74 & \\
\hline Gripe & & & & & & & & & & .74 & \\
\hline $\begin{array}{c}\text { Sensaciones de oleadas de frío o } \\
\text { calor }\end{array}$ & & & & & & & & & & .43 & \\
\hline Sensaciones de hormigueo & & & & & & & & & & & .77 \\
\hline Granos en la piel & & & & & & & & & & & .59 \\
\hline $\begin{array}{l}\text { Adormecimiento en brazos o } \\
\text { piernas }\end{array}$ & & & & & & & & & & & .48 \\
\hline
\end{tabular}

C1: Bajas defensas o problemas relacionados con tumores, C2: Problemas gastrointestinales, C3: Síntomas vestibulares o infecciosos con fiebre, C4: Hipertensión arterial, C5: Cardiorrespiratorios y estomacales, C6: Dermatológicos y genitourinarios, C7: Neurológicos o reumatoides, C8: Cardiovasculares, C9: Alergias, C10: Infecciones en vías respiratorias altas y $\mathrm{C} 11$ : Problemas circulatorios y en la piel probablemente relacionados con exceso de grasas en la dieta. 
Tabla 7

Matriz factorial rotada en la muestra de participantes tipo Ego(+)

\begin{tabular}{|c|c|c|c|c|c|c|c|c|c|}
\hline Síntomas somáticos & $\mathrm{C} 1$ & $\mathrm{C} 2$ & C3 & $\mathrm{C} 4$ & $\mathrm{C} 5$ & C6 & $\mathrm{C7}$ & $\mathrm{C} 8$ & $\mathrm{C9}$ \\
\hline Sensación de hormigueo & .83 & & & & & & & & \\
\hline Inflamación de articulaciones & .76 & & & & & & & & \\
\hline Dolor muscular & .65 & & & & & & & & \\
\hline Dolor de espalda & .63 & & & & & & & & \\
\hline Molestias en oídos & .62 & & & & & & & & \\
\hline Temblor muscular & .58 & & & & & & & & \\
\hline Dolores en la nuca o zona cervical & .57 & & & & & & & & \\
\hline Dolor de articulaciones & .50 & & & & & & & & \\
\hline Contracciones o sacudidas musculares & .49 & & & & & & & & \\
\hline Malestar al orinar o después & & .92 & & & & & & & \\
\hline Molestias en zonas genitales & & .89 & & & & & & & \\
\hline Dolor en zonas genitales & & .83 & & & & & & & \\
\hline Dificultades de coordinación & & .75 & & & & & & & \\
\hline Adormecimiento en brazos o piernas & & .59 & & & & & & & \\
\hline Dolor en el pecho & & .51 & & & & & & & \\
\hline Mareos & & .41 & & & & & & & \\
\hline Catarro & & & .89 & & & & & & \\
\hline Gripe & & & .80 & & & & & & \\
\hline Tos repetitiva & & & .58 & & & & & & \\
\hline Respiración dificultosa o molesta & & & .58 & & & & & & \\
\hline Estornudos repetitivos & & & .55 & & & & & & \\
\hline Eccema o problemas semejantes en la piel & & & & .84 & & & & & \\
\hline Vómitos & & & & .84 & & & & & \\
\hline Torpeza en las manos & & & & .75 & & & & & \\
\hline Picor en zonas genitales & & & & .56 & & & & & \\
\hline Nauseas o sensación de vómitos & & & & .47 & & & & & \\
\hline Vértigos & & & & .45 & & & & & \\
\hline Herpes & & & & .41 & & & & & \\
\hline Fatiga sin causa aparente & & & & & .92 & & & & \\
\hline Sensación general de debilidad & & & & & .79 & & & & \\
\hline Estado de baja energía & & & & & .76 & & & & \\
\hline Conjuntivitis alérgica & & & & & & .77 & & & \\
\hline Picor en los ojos de tipo alérgico & & & & & & .74 & & & \\
\hline Acidez de estómago & & & & & & .60 & & & \\
\hline Picores en la nariz de tipo alérgico & & & & & & .50 & & & \\
\hline Gastritis & & & & & & .49 & & & \\
\hline Malestar estomacal & & & & & & .48 & & & \\
\hline Constipado o congestión de tipo alérgico & & & & & & .41 & & & \\
\hline
\end{tabular}


Tabla 7. (Cont.)

Matriz factorial rotada en la muestra de participantes tipo Ego(+)

\begin{tabular}{|c|c|c|c|c|c|c|c|c|c|}
\hline Síntomas somáticos & $\mathrm{C} 1$ & $\mathrm{C} 2$ & C3 & $\mathrm{C} 4$ & C5 & C6 & $\mathrm{C} 7$ & C8 & C9 \\
\hline Fibroma o tumor nuevos o que reaparecen & & & & & & & .75 & & \\
\hline Respiración dificultosa acompañada de tos & & & & & & & .74 & & \\
\hline Sudoración excesiva & & & & & & & .68 & & \\
\hline Sangre en la orina & & & & & & & .58 & & \\
\hline Dificultad al orinar & & & & & & & & .87 & \\
\hline Granos en la piel nuevos o que reaparecen & & & & & & & & .81 & \\
\hline Dolor en el pecho & & & & & & & & .54 & \\
\hline Ligero dolor en el pecho al esforzarse & & & & & & & & .51 & \\
\hline Ulceraciones o llagas & & & & & & & & & .89 \\
\hline Tensión arterial ligeramente alta & & & & & & & & & .85 \\
\hline $\begin{array}{c}\text { Necesidad de hacer dieta para tensión } \\
\text { arterial }\end{array}$ & & & & & & & & & .79 \\
\hline
\end{tabular}

C1: Dolores músculo-esqueléticos y reumatoides, C2: Síntomas genitourinarios y neurológicos (histéricos), C3: Padecimientos del sistema respiratorio, C4: Síntomas dermatológicos y vasovagales o vestibulares, C5: Cansancio/baja energía, C6: Síntomas alérgicos y estomacales, C7: Síntomas causados por tumores, C8: Síntomas relacionados con hiperactividad simpática y C9: Hipertensión arterial.

\section{Discusión}

En primer lugar se procede a discutir los aspectos de presencia de síntomas en la muestra y, retomando los objetivos del estudio, se prosigue con la relación de los síntomas con el tipo de personalidad (ego+ y ego-) y con el género.

\section{Síntomas somáticos en la muestra}

En esta investigación se observa que las personas que acuden a consulta externa del Sistema de Salud Estatal de la Ciudad de Toluca, Estado de México, reportan padecer síntomas con baja frecuencia en el intervalo del último año, dominando aquéllos que afectan al estómago, seguido de resfriados y gripes, así como dolores de cabeza, espalda y musculares. La agrupación de síntomas del análisis factorial sobre todo refleja cuadros catarrales, gripales o alérgicos. No obstante, en la naturaleza de los diversos síntomas subyacen los hábitos de salud y estilo de vida como agentes causales presentes, los cuales están íntimamente relacionados con la personalidad; asimismo, destaca el estrés y otros estados emocionales negativos que alteran el equilibrio vegetativo si son intensos y sostenidos en el tiempo. Como indican Moral (2010) y González y Landero (2006), las causas de las enfermedades que usualmente padecemos son conocidas, a pesar de ello siempre se entrelazan con aspectos de hábitos de salud, afectos negativos, rasgos de personalidad, creencias, actitudes y refuerzos ambientales, como son los síntomas antes mencionados, donde estos factores pueden tomar mayor primacía.

\section{Síntomas somáticos y estilo de personalidad}

De acuerdo con los resultados obtenidos, se observa que existen diferencias y una propensión a padecer ciertos síntomas somáticos dependiendo del tipo de tendencia de personalidad que se posea, ya sea que pertenezca al tipo ego(+) o al tipo ego(-). Estos 
tipos de tendencias, al ser estrategias de vida, llevan a la persona a competir o rendirse, a controlar o tolerar y a rechazar o aceptar, lo cual condicionará la aparición de efectos tanto biológicos como psicosocioculturales (Valdez, 2009). Se esperaba que las personas con una tendencia de personalidad ego(-), al mostrarse más débiles, tiendan a reprimirse más y a no expresarse, por lo tanto estarían más expuestas al decaimiento, a la depresión e indefensión. Asimismo, a este tipo de personalidad le atrae que le reconozcan la fuerza que tiene para soportar la adversidad, el sufrimiento y el dolor, dependiendo así de los demás; estas personas necesitan ser apoyadas, ayudadas, protegidas, queridas 0 aceptadas, siendo usualmente más sintomáticas. En congruencia con esta expectativa los resultados muestran que estas personas tienden a padecer más síntomas somáticos que las personas con una tendencia de personalidad ego(+), sobre todo de naturaleza gastrointestinal, inmunológica, genitourinaria, hipertensiva, alérgica e incluso histéricoconversiva. Estos síntomas se relacionan con un sistema inmunológico comprometido por los altos niveles de hormonas del estrés, como cortisona y adrenalina (Eysenck, 2000)

Sin embargo, las diferencias de medias encontradas no son abundantes entre los tipos de tendencia de la personalidad ego(+) y ego(). Sólo nueve de los 80 síntomas somáticos resultan diferenciales, así como dos de los ocho componentes factoriales. Debido a este resultado congruente, pero pobre, en el perfil diferencial se realiza el análisis factorial para saber si los ítems se agrupan de manera diferente para cada tipo de personalidad. Por una parte, el mayor número de factores e ítems con saturaciones altas indica una mayor definición de las constelaciones de síntomas somáticos en los participantes con tendencia de personalidad ego(-)en comparación con los participantes con tendencia de personalidad ego(+). Por otra parte, se encontró que las personas con tipo ego(-) muestran mayor definición de síntomas relacionados, primero, con el sistema inmunológico, tumores e infecciones; segundo, con el sistema gastrointestinal, después seguirían otros grupos sintomáticos. Por el contrario, las personas con un estilo ego(+) muestran mayor definición de síntomas musculoesqueléticos, seguidos por los relacionados con padecimientos genitourinarios y neurológicos, y de ahí se definen otros grupos sintomáticos. Así, los dos primeros factores con más peso reflejan un perfil diferencial de pasividad para el tipo ego(-) versus actividad para el tipo ego(+), que es congruente con la expectativa.

El desarrollar un estilo ego(+) o un ego(-) mantiene a la persona, de manera permanente, en una situación de necesidad o carencia que no es aceptada abiertamente, donde hay queja, resentimiento, insatisfacción, rencor, etcétera; por lo cual, estas tendencias llevan a tolerar o a controlar, en los resultados que se obtienen no prevalecen los buenos niveles de salud mental y física, siendo lo ideal un tipo ego(0) que alcanza la paz y el equilibrio y por consiguiente logra liberarse de los síntomas psicopatológicos y minimizar los somáticos (Valdez, 2009). No obstante, en este estudio no se consiguió completar un grupo equivalente de participantes ego(0) para contrastar toda la teoría, para lo cual se requiere continuar con la estrategia de muestreo intencional, como la descrita en el apartado de procedimiento.

\section{Síntomas somáticos y género}

Con respecto al análisis realizado entre hombres y mujeres, también se encontraron algunas diferencias significativas. Según Moral et al. (2010), la somatización parece ser más común en las mujeres que en los hombres, habiendo claras diferencias cualitativas. Del mismo modo González y Landero (2006) reportan que las mujeres presentan mayor nivel de estrés y tendencia somatizadora que los hombres. En nuestro estudio se encontró que las mujeres tienden a presentar mayor-mente síntomas somáticos, como dolor de cabeza, sensaciones de oleadas de frío o calor, colitis, dolores en la nuca o zona cervical, calambres o dolor en piernas, estreñimiento, fatiga sin causa aparente y prurito ocular de tipo alérgico. Por el contrario, los hombres manifiestan padecer con más frecuencia diarrea y vómitos. En este perfil diferencial influyen aspectos fisiológicos del ciclo menstrual, así como hábitos de salud relacionados con la dieta e ingesta de alcohol por parte de los hombres. 
En la literatura se argumenta que las mujeres manifiestan una mayor tendencia a la expresión afectiva y la mentalización de los conflictos, por lo que sufren más de ansiedad y depresión pero menos de trastorno de control de impulsos y somatizaciones en comparación con los hombres (Medina-Mora et al., 2003; Valdez, Díaz-Loving, \& Pérez, 2006). Estos datos, junto con otros estudios (Orejudo \& Froján, 2005; Moral, 2010; Moral et al., 2010), ponen de manifiesto una mayor tendencia a la somatización de las mujeres, la cual se puede atribuir al nerviosismo y el afecto negativo, más prevalente en ellas y tienen menor peso el aspecto de la mentalización o la dificultad para identificar y expresar emociones (alexitimia).

Tanto por el tipo de tendencia de personalidad como por el género existe una inclinación, a presentar síntomas somáticos, muy relacionada con alteraciones emocionales y el estrés (Delgado, 2001); no obstante, en esta muestra no se presentaron interacciones significativas entre el género y el tipo de personalidad. Por el tipo de diseño de la muestra no se pudo contrastar un dominio de la personalidad tipo ego(-) en mujeres y del tipo ego(+) en hombres, aunque debe señalarse que el sesgo de género hacia un estilo de personalidad no es muy marcado (Valdez, 2009).

\section{Discusión}

Con la limitación de una muestra de carácter intencional extraída en México, la naturaleza de autoinforme de los datos y el carecerse de un tercer grupo de personalidad tipo(0) para evaluar en su totalidad la teoría de Valdez (2009), se concluye que existe un perfil diferencial de síntomas somáticos entre las tendencias de personalidad ego(-) y ego(+), la primera con tendencia más sintomática; las diferencias aparecen en síntomas inmunológicos, gastrointestinales, alérgicos, hipertensivos y renales. Con base en la interrelación dentro de cada muestra de tipo de personalidad, los síntomas que involucran al sistema inmune y los gastrointestinales se definen más en el tipo ego(-) y los músculo-esqueléticos y genitourinarios en el tipo ego(+). También hubo diferencias por género, siendo las mujeres más sintomáticas, aunque el género y la tendencia de personalidad no interactúan. Sólo la diarrea y los vómitos se manifestaron más en los hombres. Dolores en nuca, cuello y cabeza, fatiga, calambres, alteraciones en el tránsito intestinal, prurito ocular alérgico, así como oleadas de frío y calor son más frecuentes en mujeres.

Por lo tanto, podemos afirmar que los tipos de personalidad y el género, como factores se-parados, podrían permitir el diseño e implementación de estrategias diferenciales de intervención en síntomas somáticos, resultando útil en la evaluación de estos tipos de personalidad el cuestionario de Valdez (2008). Con estos datos se busca fomentar el estudio de la Teoría del Equilibrio y la Paz contemplada. 


\section{Referencias}

Cardenal, V., Ortiz, M., \& Martín, I. (2008). Life stressors, emotional avoidance and Breast Cancer. The Spanish Journal of Psychology, 11(2), 522-530.

Davis, R. (2009). Health psychology: stress, coping, and well-being. En S. R. Feldman (Ed.), Understanding psychology (9th edition, pp. 334-357). New York, NY: McGraw Hill.

Delgado, A. (2001). Salud y género en las consultas de atención primaria. Atención Primaria, 27(2), 75-78.

Eysenck, H. J. (2000). Personality and risk factor in cancer and coronary heart disease. En D. T. Kenny, J. G. Carlson, F. J. McGuigan, \& J. L. Sheppard (Eds.), Stress and health: Research and clinical applications (pp. 291-318). Amsterdam: Arwood Academic Publishers.

Folkman, S. (2011). The Oxford handbook of stress, health, and coping. New York: Oxford University Press.

Fritz, G. K., Fritsch, S., \& Hagino, O. (1997). Samatoform disorders in children and adolescents: a review of the past 10 years. Journal of the American Academy of Child and Adolescent Psychiatry, 36(10), 1329-1338.

González, M. T., \& Landero, R. (2006). Síntomas psicosomáticos y teoría transaccional del estrés. Ansiedad y Estrés, 12(1), 45-61.

Hernández, J. M. (2000). La personalidad. Elementos para su estudio. Madrid, España: Editorial Biblioteca.

Hiller, W., Fichter, M. M., \& Rief, W. A. (2003). Controlled treatment study of somatoform disorders including analysis of healthcare utilization and cost-effectiveness. Journal of Psychosomatic Research, 54(4), 369-380.
Kassinove, H. (Ed.) (1995). Anger disorders: Definitions, diagnosis and treatment. Washington, DC: Taylor y Francis International Publishers.

Lazarus, R. S. (1999). Stress and emotion. A new synthesis. NY: Springer.

Lipowski, Z. J. (1988). Somatization. The concept and its clinical application. American Journal of Psychiatry, 145, 1358-1368.

Lobo, A., García, J., Campos, R., Marcos, G., \& Pérez, M. (1996). Somatization in primary care in Spain: I. Estimates of prevalence and clinical characteristics. Working Group for the Study of the Psychiatric and Psychosomatic Morbidity in Zaragoza. The British Journal of Psychiatry, 168, 344-348.

Medina-Mora, M. E., Borges, G., Lara, C., Benjet, C., Blanco, J., Fleiz, C., Villatoro, J., Rojas, E., Zambrano, J., Casanova, L., \& Aguilar-Gaxiola, S. (2003). Prevalencia de trastornos mentales y usos de servicios: resultados de la encuesta nacional de epidemiología psiquiátrica en México. Salud Mental, 26(4), 1-16.

Moral, J. (2006). Análisis factorial y su aplicación al desarrollo de escalas. En R. Landero \& M. T. González (Ed.), Estadística con SPSS y metodología de la investigación (pp. 387443). México: Trillas.

Moral, J. (2010). Modelos para explicar la somatización en parejas casadas. En J. Moral, M. T. González y R. Landero (Eds.), Evaluación, modelos y tratamiento de la somatización. Su aplicación al tratamiento de la fibromialgia (pp. 131-180). México, DF: Editorial Plaza y Valdés y UANL.

Moral, J., González, M. T., \& Landero, R. (2011). Estrés percibido, ira y burnout en amas de casa mexicanas. Revista Iberoamericana de Psicología y Salud, 2(2), 139-153.

Moral, J., Valdez, J. L., González-Arratia, N. I., González, S., Alvarado, B. G., Soraya, L., \& Goana, L. S. (2010). Emociones y síntomas 
físicos medicamente no explicados en mujeres y hombres adultos. Medicina Universitaria, 12(47), 11-22.

Orejudo, S. O., \& Froján, M. X. (2005). Síntomas somáticos: predicción diferencial a través de variables psicológicas, sociodemográficas, estilo de vida y enfermedades. Anales de Psicología, 21(2), 276-285.

Sandín, B., \& Chorot, P. (1995). Escala de Síntomas Somáticos Revisada (ESS-R). Madrid: UNED.

Spielberger, Ch. D., \& Moscoso, M. S. (1996). Reacciones emocionales del estrés: ansiedad y cólera. Avances en Psicología Clínica Latinoamericana, 14, 59-81.

Valdez, J. L., Díaz-Loving, R., \& Pérez, M. (2006). Los hombres y las mujeres en México: dos mundos distantes y complementarios. Toluca, Estado de México: Universidad Autónoma del Estado de México.

Valdez, J. L. (2008). La evaluación de nuestras tendencias de personalidad: hacia el autoconocimiento para una vida en armonía. En J. Moral (Ed.), Investigaciones en psicología social, personalidad y salud (pp. 72-89). México, DF: CUMEX.

Valdez, J. L. (2009). Teoría de la Paz o Equilibrio: una nueva teoría que explica las causas del miedo y del sufrimiento, y que nos enseña a combatirlos. México, DF: EDAMEX.

Vallejo, J. (2003). Introducción a la psicopatología y a la psiquiatría (5ta edición). Barcelona, España: Masson.

Woolfolk, R. L., \& Allen, L. A. (2007). Treating somatization. A cognitive-behavioral approach. New York, NY: The Guilford Press. 